GIL, P.T.; FONTES, P.C.R.; CECON, P.R.; FERREIRA, F.A. Índice SPAD para o diagnóstico do estado de nitrogênio e para o prognóstico da produtividade da batata. Horticultura Brasileira, Brasília, v. 20, n. 4, p. 611-615, dezembro 2002.

\title{
Índice SPAD para o diagnóstico do estado de nitrogênio e para o prog- nóstico da produtividade da batata
}

\author{
Paula Torrealba de Gil ${ }^{1}$; Paulo Cezar Rezende Fontes ${ }^{2}$; Paulo Roberto Cecon ${ }^{3}$; Francisco Affonso \\ Ferreira $^{4}$ \\ 'UPEL-IPR "El Mácaro”. Subdirección Investigación y Postgrado. Carretera Nacional Maracay-Turmero (2114), Sector El Mácaro, Edo. \\ Aragua, Apartado Postal 053. Venezuela. ${ }^{2,4}$ UFV, Depto. Fitotecnia, 36.570-000 Viçosa-MG; pacerefo@mail.ufv.br. ${ }^{3} \mathrm{UFV}$, Depto. \\ Informática, 36.570-000 Viçosa-MG.
}

\section{RESUMO}

Determinou-se o nível crítico do índice SPAD (Soil Plant Analysis Development) na folha da batateira e verificou-se a possibilidade de sua utilização no prognóstico da produção de tubérculos de batata, cultivar Monalisa. O experimento foi realizado em Argissolo Vermelho-Amarelo, com o teor de $\mathrm{N}$ mineral de $39 \mathrm{mg}$ $\mathrm{kg}^{-1}$. O ensaio foi conduzido no delineamento em blocos ao acaso, com quatro repetições, em esquema de parcela subdividida. As parcelas foram constituídas por cinco doses de $\mathrm{N}(0 ; 25 ; 50 ; 100$ e 200 $\mathrm{kg} \mathrm{ha}^{-1}$ ) aplicadas nos sulcos em pré-plantio. As subparcelas constituíram as quatro doses de $\mathrm{N}\left(0 ; 90 ; 180\right.$ e $\left.360 \mathrm{~kg} \mathrm{ha}^{-1}\right)$, aplicadas em cobertura, $10 \mathrm{~cm}$ ao lado das plantas, aos 23 dias após a emergência (DAE), imediatamente antes da amontoa. Foi utilizado o sulfato de amônio. Foram feitas medições do índice SPAD no folíolo terminal da quarta folha a partir do ápice (QF) em seis épocas. A primeira (SPAD1) foi realizada três dias antes da adubação em cobertura e as demais aos 7; 17; 27; 37 e 47 dias após a aplicação de $\mathrm{N}$ em cobertura. Também foram determinados os índices SPAD em folhas mais velhas (FM) que a $\mathrm{QF}$. Na primeira época foram determinados os teores de N na QF. O índice SPAD1 e o teor de N aumentaram de forma quadrática em função das doses de $\mathrm{N}$ em pré-plantio, atingindo os níveis críticos de 44,9 unidades e de 6,2 dag.kg-1, respectivamente. Houve correlação linear e positiva entre os valores do índice SPAD1 e os da produção de tubérculos, indicando que o índice SPAD pode ser usado no prognóstico da produtividade da cultura. Em todas as épocas de amostragens, os índices SPAD na QF e na FM foram influenciados pelas doses de $\mathrm{N}$ porém, atingiram valores diferentes, mostrando a necessidade de padronizar a época e a folha a ser amostrada.

Palavras-chave: Solanum tuberosum, clorofila, tubérculos, nível crítico.

\section{ABSTRACT \\ SPAD index for nitrogen status diagnosis and potato yield prognosis}

The SPAD critical level (Soil Plant Analysis Development) in potato leaves was established to verify the possibility of its utilization in Monalisa potato tuber yield prognosis. The experiment was installed in a Red-Yellow Argisoil having $39 \mathrm{mg} \mathrm{kg}^{-1}$ of mineral nitrogen content. The experiment was laid out in randomized complete blocks with split plot design and four replications. In the plot were set five $\mathrm{N}$ rates $\left(0 ; 25 ; 50 ; 100\right.$ and $\left.200 \mathrm{~kg} \mathrm{ha}^{-1}\right)$, applied in the furrows before planting. In the split plot were set four side-dressed $\mathrm{N}$ rates $\left(0 ; 90 ; 180\right.$ and $\left.360 \mathrm{~kg} \mathrm{ha}^{-1}\right)$, applied in bands $10 \mathrm{~cm}$ apart plant side at 23 days after plant emergence, before hilling. SPAD indices were obtained from the terminal leaflet of the fourth leaf from the apex $(\mathrm{QF})$ for six times. The first one (SPAD1) was measured three days before the side-dress $\mathrm{N}$ fertilization and the others at $7 ; 17 ; 27 ; 37$ and 47 days after the side-dress $\mathrm{N}$ application. SPAD indices were also determined in leaves older (FM) than the QF. N content in the QF was determined at the first sampling time. SPAD1 index and $\mathrm{N}$ content in the QF increased as preplanting $\mathrm{N}$ rates increased following a quadratic function, attaining the critical values of 44.9 and $6.15 \mathrm{dag} . \mathrm{kg}^{-1}$, respectively. The correlation between SPAD1 indices and tuber yield was linear and positive, showing that SPAD1 could be used as a prognostic index of potato yield. At all sampling dates, SPAD indices in the QF and FM leaves were affected by $\mathrm{N}$ rates but they reached different values, showing the necessity to standardize the time and leaf to be sampled.

Keywords: Solanum tuberosum, chlorophyll, tuber, critical level.

(Recebido para publicação em 18 de abril de 2001 e aceito em 13 de setembro de 2002)

\begin{abstract}
$\mathrm{A}^{\mathrm{s}}$ juste no programa de fertilização nitrogenada de uma cultura pode ser conseguido por correto monitoramento e diagnóstico do estado nutricional das plantas (Huett et al., 1997) que envolve tradicionalmente a análise dos teores de $\mathrm{N}$ na matéria seca de folhas e posterior interpretação dos resultados. Porém, a utilização da análise foliar apresenta limitações, como o
\end{abstract}

tempo gasto entre a tomada das amostras e a obtenção dos resultados. Atualmente, como parte da agricultura de precisão, é necessário o sensoriamento em tempo real do estado de nutrientes da planta. Dentre as técnicas mais recentes com potencial para avaliar o estado de nitrogênio da planta em tempo real destaca-se a análise da intensidade do verde das folhas, pelo fato de haver cor- relação significativa entre a intensidade do verde e o teor de clorofila com a concentração de $\mathrm{N}$ na folha. Em doze espécies de plantas, Marquard \& Tipton (1987) observaram correlação positiva entre a intensidade do verde e o teor de clorofila das folhas e Guimarães et al. (1999) encontraram correlação entre o teor de clorofila e a concentração de nitrogênio nas folhas do tomateiro.

2, 3, 4 Bolsistas do CNPq. 
A avaliação do verde da folha de forma rápida e com baixo custo tornouse mais fácil com os recentes avanços e aperfeiçoamento dos medidores portáteis (Blackmer \& Schepers, 1995; Guimarães et al., 1999) possibilitando a sua utilização como critério de avaliação do estado de nitrogênio das plantas. Um destes medidores portáteis é o SPAD502 (Soil Plant Analysis Development), que apresenta facilidade de operação, permite avaliações in situ e que pode assim ser utilizado como ferramenta auxiliar na tomada de decisão sobre a adubação nitrogenada. O instrumento SPAD-502 avalia, quantitativamente, a intensidade do verde da folha, medindo as transmissões de luz a $650 \mathrm{~nm}$, onde ocorre absorção de luz pela molécula de clorofila e a $940 \mathrm{~nm}$, onde não ocorre absorção. Com estes dois valores, o equipamento calcula um número ou índice SPAD que, normalmente, é altamente correlacionado com o teor de clorofila da folha (Markwell et al., 1995; Guimarães et al., 1999) e pode identificar deficiência de $\mathrm{N}$ além de ter potencial de identificar situações onde a aplicação adicional de N não seja necessária.

Indices SPAD obtidos em folhas de diversas espécies apresentaram correlação positiva com a suficiência de $\mathrm{N}$ (Piekielek \& Fox, 1992; Blackmer \& Schepers, 1995; Guimarães et al., 1999; Shapiro, 1999) podendo ser considerado um índice para avaliar o estado de nitrogênio das plantas. Recentemente, foi demonstrado a potencialidade do SPAD-502 para avaliar a resposta de diversas espécies à aplicação do nitrogênio (Hoel \& Solhaug, 1998; Wu et al., 1998; Sainz-Rozas \& Echeverria, 1998; Madakadze et al., 1999; Carreres et al., 2000; Sandoval-Villa et al., 2000).

De modo similar às outras culturas, o diagnóstico do estado de $\mathrm{N}$ da batata pode ser realizado pela análise do teor de clorofila nas folhas ou a intensidade do verde utilizando o clorofilômetro portátil SPAD 502 (Vos \& Bom, 1993; Minotti et al.,1994; Rodrigues et al., 2000). Para tal, é necessário, porém, dispor do valor do nível crítico a ser usado como referência que pode variar de acordo com a espécie, época de plantio da cultura (Balasubramanian et al., 1999), com os estádios anatômico e morfológico das folhas e estádio fenológico das plantas (Paliwal \& Karunaichamy, 1995).

São inexistentes no Brasil trabalhos de pesquisas buscando o estabelecimento do valor crítico do índice SPAD em folhas de batateira em condições de campo. Assim, este trabalho teve como objetivo determinar o nível crítico do índice SPAD na folha e analisar a possibilidade de sua utilização no prognóstico da produção de tubérculos de batata.

\section{MATERIAL E METODOS}

O experimento foi realizado de 30 de março a 4 de julho de 2000, na UFV, em Argissolo Vermelho-Amarelo, com o teor inicial de $\mathrm{N}$ mineral na camada de $0-20 \mathrm{~cm}$ de profundidade de $39 \mathrm{mg}$ $\mathrm{kg}^{-1}$. Foi conduzido no delineamento de blocos ao acaso, com quatro repetições, em esquema de parcela subdividida. Nas parcelas ficaram as cinco doses de $\mathrm{N}(0$; $25 ; 50 ; 100$ e $\left.200 \mathrm{~kg} \mathrm{ha}^{-1}\right)$ que foram aplicadas nos sulcos, em pré-plantio. Nas subparcelas ficaram as quatro doses de $\mathrm{N}\left(0 ; 90 ; 180\right.$ e $\left.360 \mathrm{~kg} \mathrm{ha}^{-1}\right)$, aplicadas em cobertura, $10 \mathrm{~cm}$ ao lado das plantas, aos 23 dias após a emergência (DAE) imediatamente antes da amontoa. Foi utilizado o sulfato de amônio. As subparcelas continham 40 plantas, sendo 16 úteis na área de $3,84 \mathrm{~m}^{2}$.

O plantio da batata (Solanum tuberosum L.), cultivar Monalisa, foi feito em 30 de março, no espaçamento de $80 \mathrm{~cm}$ entre fileiras e $30 \mathrm{~cm}$ entre plantas, sendo a cultura conduzida nos moldes da região da Zona da Mata de $\mathrm{MG}$, com irrigação por aspersão. $\mathrm{O}$ ciclo da cultura foi de 80 dias após a emergência.

Ao longo do ciclo foram feitas medições do índice SPAD no folíolo terminal de seis plantas úteis da subparcela, escolhidas ao acaso, e a média representou o valor de cada unidade experimental. Para a determinação do índice SPAD foi utilizado o medidor portátil SPAD502, da empresa Minolta, seguindo os procedimentos adotados por Guimarães (1998). A primeira medição, denominada SPAD1, foi feita três dias antes da adubação nitrogenada em cobertura (ANC), na quarta folha a partir do ápice (QF) antes da mesma ser destacada da planta para ser analisada quanto ao teor de nitrogênio, pelo método de Nessler (Jackson, 1982). Posteriormente, a cada dez dias, aos 7; 17; 27; 37 e 47 dias após a ANC, foi medido o índice SPAD da QF de seis plantas da subparcela, que foram identificados como SPAD2; 3; 4; 5 e 6 , respectivamente. Também, foram determinados em três épocas os índices SPAD de uma folha marcada (FM) sinalizada com uma fita. A primeira determinação foi feita aos 27 dias após a ANC, quando a folha era a quinta a partir do ápice (SPAD4FM), a segunda (SPAD5FM) e a terceira (SPAD6FM) foram feitas na mesma folha, 10 e 20 dias após a primeira determinação.

Os valores das variáveis dependentes foram submetidos à análise de variância e metodologia de superfície de resposta. Os modelos foram escolhidos com base na significância dos coeficientes de regressão, utilizando-se o teste de t adotando-se o nível de $1\left({ }^{* *}\right)$ e $5 \%\left(^{*}\right)$ de probabilidade, no coeficiente de determinação e no fenômeno em estudo.

A partir das doses de 144,3 e 360 $\mathrm{kg} \mathrm{ha}^{-1}$ de $\mathrm{N}$ em pré-plantio e cobertura, respectivamente, que propiciaram a máxima produção de tubérculos (GIL, 2001), foram calculados os valores dos níveis críticos dos índices SPAD2 a SPAD6; os níveis críticos do índice SPAD1 e do teor de nitrogênio foram calculados com a dose de $144,3 \mathrm{~kg} \mathrm{ha}^{-1}$ de N, seguindo metodologia descrita em Fontes (2001).

Foi determinada a relação entre os teores $\mathrm{N}$ na QF e os valores dos índices SPAD1 e também destes com a produtividade dos tubérculos, em 77 pares de dados pois foram retiradas três observações por serem consideradas out lier (fora de linha), de acordo com os critérios de resíduo padronizados e da distancia de Cook (Myers \& Montgomery, 1995).

\section{RESULTADOS E DISCUSSÃO}

O índice SPAD1 e o teor de nitrogênio na quarta folha completamente expandida (QF) da batata, avaliados três dias antes da adubação com $\mathrm{N}$ em cobertura, aumentaram de forma quadrática e similarmente em função das doses de N em pré-plantio (Figura 1).

Similar tendência na variação dos dois índices indicam a possibilidade de 
utilização do valor SPAD na caracterização do estado de nitrogênio da batata. Para tal, pode ser usada a relação $\mathrm{Y}$ $=-4,124+0,229 * * X, r=0,94$, obtida no presente experimento, onde $\mathrm{Y}$ é o teor de $\mathrm{N}$ na matéria seca da $\mathrm{QF}$, expresso em dag $\mathrm{kg}^{-1}$ e $\mathrm{X}$, é o valor do índice SPAD1. Correlação positiva entre a adição de nitrogênio e o teor de clorofila na folha da batata foi obtida por Vos \& Bom (1993), indicando que o teor de clorofila na folha está relacionado com o estado de nitrogênio da planta.

No presente trabalho, o valor crítico calculado do índice SPAD1 foi 44,9 unidades. No campo, Minotti et. al. (1994) determinaram os valores variando de 49 a 56 unidades, dependendo do ano, cultivar e localidade, para o índice SPAD na quarta ou quinta folha jovem completamente expandida da batateira associados com a dose de $\mathrm{N}$ que permitiu o máximo rendimento de tubérculos comercializáveis. De modo coincidente, Malavolta et al. (1997) citam a faixa de 49-56 como a adequada para o índice SPAD na quarta ou quinta folha nova totalmente expandida da batata. Rodrigues et al. (2000) determinaram o valor de 39,6 unidades SPAD como o nível crítico associado a dose de $\mathrm{N}$ que propiciou a máxima produção de matéria seca da parte aérea da batateira em solução nutritiva, em estufa.

$\mathrm{O}$ valor crítico calculado do teor de $\mathrm{N}$ na matéria seca da QF foi 6,15 dag $\mathrm{kg}^{-1}$. Valores de 4 a $6,5 \mathrm{dag} \mathrm{kg}^{-1}$ na matéria seca da folha completamente desenvolvida de batateira jovem são citados como adequados (Mill \& Jones Jr., 1996; Huett et al., 1997; Rodrigues et al., 2000a). Na interpretação de resultados de análise foliar envolvendo o uso de nível crítico são necessárias algumas precauções (Fontes, 2001). Uma delas é considerar a(s) época(s) em que o fertilizante foi fornecido às plantas pois o parcelamento da dose acarretará modificação no valor crítico.

O SPAD2 aumentou de forma linear com o aumento das doses de $\mathrm{N}$ em pré-plantio em todas as doses de $\mathrm{N}$ em cobertura (Figura 2). O SPAD3 aumentou de forma linear devido às doses de nitrogênio em pré-plantio e de forma quadrática devido às doses de $\mathrm{N}$ em cobertura $\left(\mathrm{Y}=37,332+0,0267295^{* *} \mathrm{CO}\right.$ -

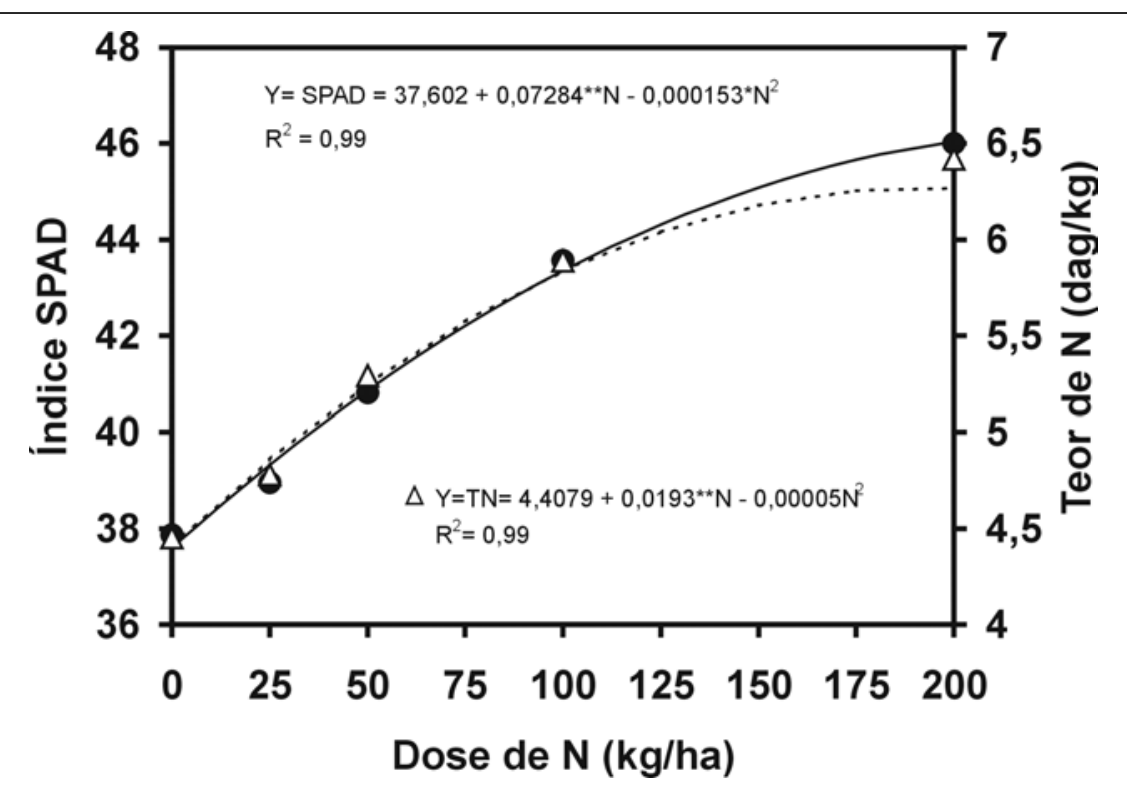

Figura 1. Estimativas do índice SPAD e do teor de nitrogênio (NT) na quarta folha da batata em função de doses de nitrogênio aplicada em pré-plantio. Viçosa, UFV, 2000.

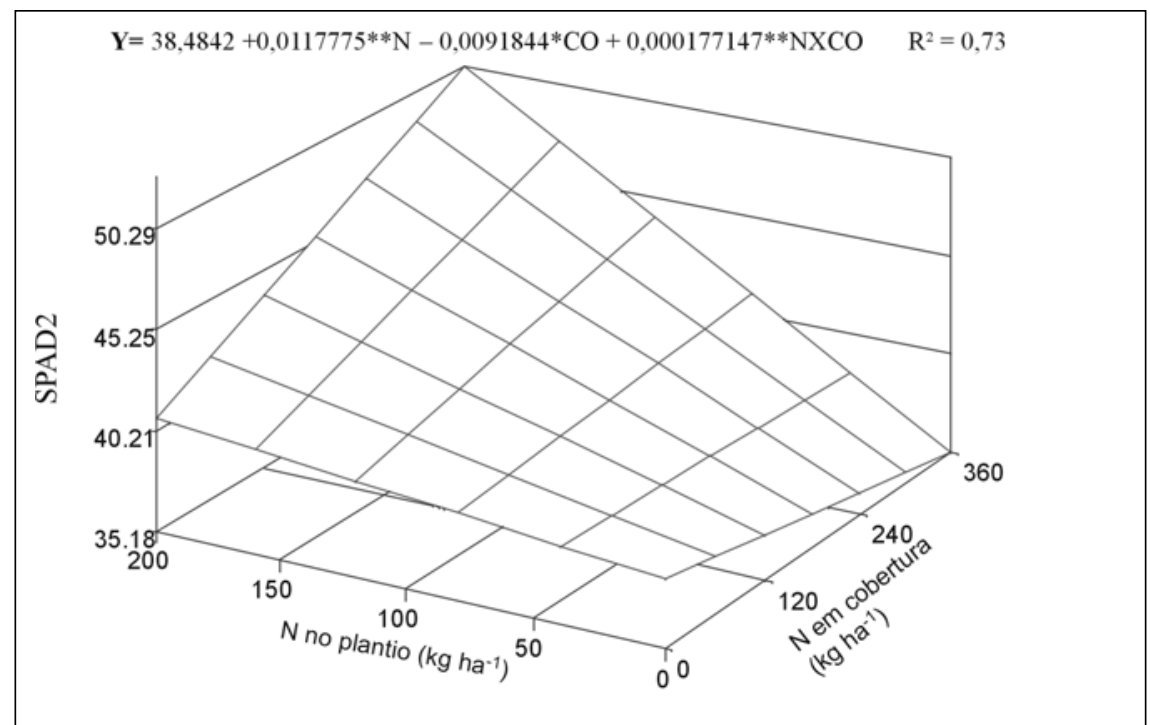

Figura 2. Indice SPAD2 da quarta folha da batateira, em função de doses de $\mathrm{N}$ aplicadas em pré-plantio $(\mathrm{N})$ e em cobertura $(\mathrm{CO})$.

$0,0000437233 * * \mathrm{CO}^{2}$ $\left.0,02501850 * * \mathrm{~N} ; \mathrm{R}^{2}=0,87\right)$ e também os valores dos índices SPAD4 $(\mathrm{Y}=39,0400+0,030315 * * \mathrm{CO}$ $0,0000435449 * \mathrm{C}^{2}+0,0198775 * * \mathrm{~N}-$ $\left.0,0000636587 * \mathrm{NxCO} ; \mathrm{R}^{2}=0,91\right)$, SPAD5 $\quad(\mathrm{Y}=34,7146 \quad+$ $0,00869905 * \mathrm{CO}+0,0138644 * * \mathrm{~N}$; $\left.\mathrm{R}^{2}=0,57\right)$ e SPAD6 $(\mathrm{Y}=34,3958+$ $0,00936667 * \mathrm{CO}+0,01935560 * * \mathrm{~N}$; $\mathrm{R}^{2}=0,38$ ) foram influenciados pelas doses de N. Os valores dos índices SPAD obtidos no presente experimento fica- ram próximos dos obtidos por Vos \& Bom (1993), em outras condições experimentais.

Com a dose de $144,3 \mathrm{~kg} \mathrm{ha}^{-1}$ de $\mathrm{N}$ aplicada em pré-plantio e zero de $\mathrm{N}$ em cobertura, o valor estimado do SPAD2 foi 40,2 que passou para 46,1 (nível crítico) com a dose de $144,3 \mathrm{~kg} \mathrm{ha}^{-1}$ de N em pré-plantio e $360 \mathrm{~kg} \mathrm{ha}^{-1}$ em cobertura (Figura 2). Portanto, sete dias após a aplicação de $360 \mathrm{~kg} \mathrm{ha}^{-1}$ de $\mathrm{N}$ em cobertura houve aumento de 5,9 unidades SPAD ou $61 \mathrm{~kg} \mathrm{ha}^{-1}$ de $\mathrm{N}$ foram neces- 
Tabela 1. Incrementos nos valores dos índices SPAD2 a SPAD6 na quarta folha causados pelas doses de nitrogênio em cobertura no tratamento que recebeu a dose ótima de nitrogênio no plantio. Viçosa, UFV, 2000.

\begin{tabular}{lcccc}
\hline & $\begin{array}{c}\text { Data da } \\
\text { Índice }\end{array}$ & \multicolumn{3}{c}{ Dose de $\mathbf{~}$ em cobertura $\left(\mathbf{k g ~ h a}^{-1}\right)$} \\
\cline { 3 - 5 } & determinação & & $\mathbf{3 6 0}$ \\
\hline SPANC) & 7 & $\mathbf{9 0}$ & $\mathbf{1 8 0}$ & 5,9 \\
SPAD3 & 17 & 1,5 & 2,9 & 4,0 \\
SPAD4 & 27 & 1,1 & 3,4 & 2,0 \\
SPAD5 & 37 & 1,6 & 2,4 & 3,2 \\
SPAD6 & 47 & 0,8 & 1,6 & 3,4 \\
\hline
\end{tabular}

$\mathrm{DANC}=$ dias após a aplicação do nitrogênio em cobertura.

sários para aumentar 1 unidade SPAD. Tal valor pode ser usado como referência na determinação da dose de $\mathrm{N}$ a ser aplicada em cobertura na cultura da batata ao ser utilizado o SPAD. Em videira, a aplicação de $50 \mathrm{~kg} \mathrm{ha}^{-1}$ de $\mathrm{N}$ modificou o valor do índice SPAD em torno de 2 unidades (Porro et al., 2001).

Com a dose de $144,3 \mathrm{~kg} \mathrm{ha}^{-1}$ de $\mathrm{N}$ aplicada em pré-plantio, em todas as épocas de determinação, os valores estimados dos índices SPAD na QF (SPAD2 a SPAD6) tiveram incrementos crescentes com o aumento da dose de $\mathrm{N}$ em cobertura (Tabela 1) indicando o aumento na intensidade do verde. Medida pelo mais acentuado incremento no índice SPAD, a mais rápida utilização do $\mathrm{N}$ foi verificada aos 17 dias após a aplicação das doses de 90 e 180 $\mathrm{kg} \mathrm{ha}^{-1}$ e aos 7 dias com a dose de 360 $\mathrm{kg} \mathrm{ha}^{-1}$ (Tabela 1) indicando maior rapidez na utilização do N com a maior dose aplicada.

$\mathrm{O}$ efeito das doses de $\mathrm{N}$ sobre os índices SPAD na folha marcada (FM) foi semelhante ao ocorrido com a QF, porém, com valores diferentes [ $\begin{array}{llllllll}\mathrm{S} & \mathrm{S} & \mathrm{P} & \mathrm{A} & \mathrm{D} & 4 & \mathrm{~F} & \mathrm{M}\end{array}$ : $\mathrm{Y}=37,4710+0,022947^{* *} \mathrm{~N}+0,019241^{* *} \mathrm{CO}$ - $\quad 0,00002397773 * \mathrm{CO}^{2} \quad$ $0,0000599214 * * \mathrm{~N}$ x C O ; $\left.\mathrm{R}^{2}=0,74\right)$;(SPAD5FM: $\mathrm{Y}=38,1279+$ $0,00610365^{* *} \mathrm{CO}+0,0113250 * * \mathrm{~N} ; \mathrm{R}^{2}=$ 0,54) e (SPAD6FM: Y=33,2343+ $\left.\left.0,0184431^{* * N} ; \mathrm{R}^{2}=0,47\right)\right]$. Portanto, tanto na folha jovem (QF) como na mais madura (FM), o índice SPAD variou em função da dose de N. Entretanto, os valores foram diferentes, podendo ser devido a remobilização do $\mathrm{N}$ nas folhas.

Com as doses ótimas de $\mathrm{N}$ no plantio e em cobertura, os níveis críticos esti- mados dos índices SPAD2 a SPAD6 na QF foram 46,1; 44,9; 43;9 e 42,6 e 40,6, respectivamente e dos SPAD4 a SPAD6 na FM foram 41,5; 42,0 e 35,9, respectivamente, indicando a necessidade de padronizar tanto a época de amostragem como a folha para determinar o nível crítico do índice SPAD em batata.

Houve correlação linear positiva e significativa entre os valores do índice SPAD1 e os da produção de tubérculos, expressa pela relação $\mathrm{Y}=-0,0866+$ $0,0152 \mathrm{X}, \mathrm{r}=0,62$, sendo $\mathrm{Y}$ a produtividade de tubérculos e X, o índice SPAD1. Os valores observados de $\mathrm{Y}$ variaram de 35 a 49 e os de $X$ variaram de 0,363 a $0,725 \mathrm{~kg} /$ planta. Em avaliação feita durante o ciclo da cultura do milho, Piekielek \& Fox (1992) observaram que os valores SPAD na folha correlacionaram-se com a quantidade de $\mathrm{N}$ adicionada e com a produção de grãos.

O coeficiente de correlação talvez fosse maior se não houvesse a incidência de Erwinia e Rhizoctonia nas plantas, antecipando o final do ciclo da cultura e prejudicando a produtividade. Mesmo assim, conclui-se ser possível utilizar o índice SPAD medido na QF, aos 20 dias após a emergência, no prognóstico da produtividade da cultura da batata.

\section{LITERATURA CITADA}

BALASUBRAMANIAN, V.; MORALES, A.C.; CRUZ, R.T.; ABDULRACHMAN, S.; LADHA, J.K.; DENNING, G.L. On-farm adaptation of knowledge-intensive nitrogen management technologies for rice systems. Nutrient Cycling in Agroecosystems, v. 53, n. 1, p. 59-69, 1999.

BLACKMER, T.M.; SCHEPERS, J.S. Use of a chlorophyll meter to monitor nitrogen status and schedule fertigation for corn. Journal Production Agriculture, v. 8, p. 56-60, 1995.
CARRERES, R.; SENDRA, J.; BALLESTEROS, R.; CUADRA, J.G. Effects of preflood nitrogen rate and midseason nitrogen timing on flooded rice. Journal of Agricultural Science, v. 134, n. 4, p. 379-390, 2000.

FONTES, P.C.R. Diagnóstico do estado nutricional das plantas. Viçosa: UFV, 2001. 122 p.

GIL, P.T. Índices e eficiência de utilização de nitrogênio pela batata influenciados por doses de nitrogênio em pré-plantio e em cobertura. Viçosa: UFV. 81 p. 2001. (Tese mestrado)

GUIMARÃES, T.G. Nitrogênio no solo e na planta, teor de clorofila e produção do tomateiro, no campo e de estufa, influenciados por doses de nitrogênio. Viçosa: UFV. 201 p. 1998. ( Tese doutorado)

GUIMARÃES. T.G.; FONTES, P.C.R.; PEREIRA, P.R.G.; ALVAREZ V., V.H.; MONNERAT, P.H. Teores de clorofila determinados por medidor portátil e sua relação com formas de nitrogênio em folhas de tomateiro cultivado em dois tipos de solo. Bragantia, Piracicaba, v. 58, n. 1, p. 209-216, 1999.

HOEL, B.O.; SOLHAUG, K.A. Effect of irradiance on chlorophyll estimation with the Minolta SPAD-502 leaf chlorophyll meter. Annals of Botany, v. 82, p. 389-392, 1998.

HUETT, D.O.; MAIER, N.A.; SPARROW, L.A.; PIGGOTT,T.J. Vegetables. In: REUTER, D.J.; ROBINSON, J.B. Eds. Plant Analysis: an interpretation manual. Second edition. Collingwood, Australia: CSIRO, 1997. p. 385-464. JACKSON, M.L. Análisis quimico de suelos. Barcelona: Ediciones Omega, S.A., 1982. 662 p.

MADAKADZE, I.C.; STEWART, K.A; MADAKADZE, R.M; PETERSON, P.R.; COULMAN, B.E.; SMITH, D.L. Field evaluation of the chlorophyll meter to predict yield and nitrogen concentration of switchgrass. Journal of Plant Nutrition, v. 22. n. 6, p. 1001-1010, 1999.

MALAVOLTA, E.; VITTI, G.C.; OLIVEIRA, S.A. Avaliação do estado nutricional das plantas. Princípios e aplicações. $2^{\mathrm{a}}$ edição. Piracicaba: Associação Brasileira para Pesquisa da Potassa e do Fosfato, 1997. 319 p.

MARKWELL, J.; OSTERMAN, J.C.; MITCHELL, J.L. Calibration of the Minolta SPAD-502 leaf chlorophyll meter. Photosynthesis Research, v. 46, p. 467-472, 1995.

MARQUARD, R.D.; TIPTON, J.L. Relatioship between extractable chlorophyll and an in situ method to estimate leaf greenness. HortScience, v. 22 , n. 6 , p. $1327,1987$. 
MILLS, H.A.; JONES, Jr.,J.B. Plant analysis handbookI II. Georgia, USA: Micromacro Publishing, 1996. 422 p.

MINOTTI, P.L.; HALSETH, D.E.; SIECZKA,

J.B. Field chlorophyll measurements to assess the nitrogen status of potato varieties. HortScience, v. 29, n. 12, p. 1497-1500, 1994.

MYERS, R.H.; MONTGOMERY, D.C. Response surface methodology: Process and product optimization using designed experiments. New York: John Wiley \& Sons, Inc. 1995. 700 p.

PALIWAL, K.; KARUNAICHAMY, K.S.T.K. Insitu estimation of leaf chlorophyll by light transmittance in vegetable crops. Indian Journal Agricultural Science, v. 65, n. 5. p. 361-362, 1995.

PIEKIELEK, W.P.; FOX, R.H. Use of a chlorophyll meter to predict sidedress nitrogen requirements for maize. Agronomy Journal, v. 84, n. 59-65, 1992.
PORRO, D.; BERTAMINI, M.; DORIGATTI, C.; STEFANINI, M.; CESCHINI, A. Lo SPAD nella diagnosi dello stato nutrizionale della vite. Informatore Agrario, v. 57, n. 26, p. 49-55. 2001 RODRIGUES, F.A.; FONTES, P.C.R.; MARTINEZ, H.E.P.; PEREIRA, P.R.G. Nível crítico do índice SPAD na folha da batateira, em solução nutritiva. Horticultura Brasileira, Brasília, v. 18 , p. 764-765, 2000.

RODRIGUES, F.A.; FONTES, P.C.R.; PEREIRA, P.R.G.; MARTINEZ, H.E.P. Crescimento e teor crítico de $\mathrm{N}_{-} \mathrm{NO}_{3}$ na seiva da batateira cultivada em solução nutritiva. Horticultura Brasileira, Brasília, v.18, p. 766-767. 2000a.

SAINZ-ROZAS, H.; ECHEVERRIA, H.E. Relacion entre las lecturas del medidor de clorofila (Minolta SPAD 502) en distintos estadios del ciclo del cultivo de maiz y el rendimento en grano. Revista de la Facultad de Agronomia la Plata, v. 103, n. 1, p. 37-44, 1998.
SANDOVAL-VILLA, M.; GERTAL, E.A.; WOOD, C.W. Tomato leaf chlorophyll meter readings as affected by variety, nitrogen form, and nighttime nutrient solution strength. Journal of Plant Nutrition, v. 23. n. 5, p. 649-661, 2000.

SHAPIRO, C.A. Using a chlorophyll meter to manage nitrogen applications to corn with high nitrate irrigation water. Communications Soil Science Plant Analysis, v. 30, n. 7/8, p. 1037-1049, 1999.

VOS, J.; BOM, M. Hand-held chlorophyll meter: a promising tool to asses the nitrogen status of potato foliage. Potato Research, v. 36, p. 301-308, 1993.

WU, F.B.; WU, L.H.; XU, F.H. Chlorophyll meter to predict nitrogen sidedress requirements for short-season cotton (Gossypium hirsutum L.). Field Crops Research, v. 56, n. 3, p. 309-314, 1998. 